\title{
FORMAS DE APROPIACIÓN DEL DISCURSO SOBRE COMPETENCIAS POR PARTE DE LOS PROFESORES DE CIENCIAS NATURALES \\ -Un estudio desde el pensamiento de los profesores-
}

\author{
Chona, G.; Arteta, J.; Fonseca G.; Martínez, S.
}

\begin{abstract}
This research project recognizes the teacher as an active subject who reconstructs the knowledge and pretends to identify the conceptions about scientific competencies that the teachers have. The research considers from this perspective that the teachers show and construct a new mean/ng of be/ng a sc/ence teacher through a team work. The teachers in this research have a protagonic role and through discussions and debates which are taped and then analyzedbymeans of a cooperative worlç they get the construction of the meaning of the teaching of science in our country and show the ways to inculcate values that can and have to be developed through the teaching of experimental science.
\end{abstract}

\section{RESUMEN}

El proyecto de investigación parte de reconocer al maestro como un activo sujeto reconstructor de saber y pretende identificar las concepciones que acerca de competencias científicas poseen los profesores de ciencias experimentales. Desde esta perspectiva la investigación asume: que es desde la construcción colectiva, donde los maestros explicitamos y construimos los nuevos sentidos de la escuela, de ser maestro y de la enseñanza de las ciencias. Los maestros entonces, cobran un papel protagónico y a través de conversatorios, talleres y debates, los cuales son registrados e interpretados conjuntamente, se logra la construcción del significado respecto a la enseñanza de las ciencias en nuestro país y la definición de cuáles son las competencias científicas y de qué manera la formación en valores, pueden y deben ser desarrolladas a través de la enseñanza de las ciencias experimentales.

\section{INTRODUCCIÓN}

Somos conscientes que la sociedad colombiana, dados los altos índices de violencia que se expresan en un sinnúmero de conflictos, demanda al sistema educativo la formación de ciudadanos autónomos, conscientes y críticos. Lo anterior es posible lograrlo de mejor manera, si los educadores están preparados para asumir los nuevos retos y dispuestos trabajar desde el interior mismo de las escuelas y de las áreas de estudio que enseñan.

Por otro lado, las nuevas políticas de globalización, han llevado al sistema educativo a trabajar en la idea de mejorar la calidad de la educación a través del desarrollo de competencias básicas, lo cual ha sido un criterio en la estructuración de pruebas

\footnotetext{
* Universidad Pedagógica Nacional. Departamento de Biología y Doctorado en Educación. Calle $72 \mathrm{~N}^{0} 11$ 86. Apartado Aéreo 75144. Fax 217 3321. Santafé de Bogotá. Colombia. E-mail: arteta@uni.pedagogica.edu.co
} 
diagnósticas censales y el Examen de Estado para el ingreso a la Educación Superior. Ahora bien, dada la poca experiencia de trabajo en esta perspectiva y la gran expectativa que esto ha generado en los maestros, respeto al tipo de reorientaciones que ello implicaría en el abordaje de las diferentes áreas de enseñanza, obliga a hacer una revisión y análisis sobre cómo desarrollar y cuáles competencias propiciar tanto en los estudiantes como en los profesores.

Trabajos de investigación anteriores (Chona et. al., 1998; Reyes et. al., 1999), sobre el pensamiento de los profesores de ciencias en Colombia, han empezado a documentar las características de los diversos ambientes de formación personal y científica que los profesores de ciencias ofrecen a sus estudiantes, así como también algunas dificultades de los docentes, en la introducción de conceptos y manejo de proyectos de manera sistemática.

De igual manera, el trabajo educativo orientado a la formación en valores es apreciado y reconocido por los docentes, pero falta organización de prácticas que desde las distintas áreas propendan al desarrollo humano integral, que tomen como principios el respeto a la vida, al otro y a los derechos humanos.

\section{CONTEXTO DE LA INVESTIGACIÓN}

Esta investigación parte de reconocer a los docentes como activos sujetos reconstructores de saberes no sólo científicos sino también pedagógicos, que se hacen explícitos durante el desarrollo de sus acciones educativas. Por ello, creemos que es necesario recuperar las distintas experiencias, que los maestros de ciencias experimentales han puesto en práctica, respecto a la incorporación del espíritu científico y la formación en valores en sus estudiantes. A partir de lo anterior y de la reflexión fundamentada, podemos construir sentidos en torno a lo que podría ser una propuesta para articular el necesario desarrollo de competencias científicas básicas, con una sólida formación en valores, que propendan por una convivencia pacífica y solidaria.

Atendiendo a los resultados de trabajos anteriores del grupo de investigación, nos preguntamos: ¿De qué manera joque hoy conocemos del pensamiento del profesor contribuye a comprender la complejidad de las nuevas tareas que se quieren asumir? Consideramos entonces, que se hace necesario adelantar investigaciones que exploren y sistematicen el pensamiento del profesor de ciencias experimentales en torno al actual enfoque de trabajo en competencias, puesto que de él dependen, en gran medida, las reales posibilidades de la puesta en marcha de los urgentes cambios y reorientaciones que requiere la educación en ciencias experimentales en nuestro país.

Con tal propósito nos planteamos las siguientes preguntas orientadoras, para las necesarias búsquedas y actividades que adelantaremos con los maestros:

- ¿Qué vamos a hacer los maestros ante tantos nuevos aspectos, (información, métodos, inteligencias múltiples, logros, competencias, constructivismo, postmodernismo, globalización) a tener en cuenta para la educación?

- ¿De qué manera lo que sabemos los profesores de ciencias naturales contribuye a abordar los cambios educativos y cualificar la enseñanza de la Biología? 
- ¿Cuáles son las concepciones, que acerca de las competencias científicas, poseen los profesores de ciencias experimentales para orientar la formación de los estudiantes?

- ¿Cuál sería la estructura valorativa apreciada por los profesores de ciencias experimentales en nuestra sociedad?

- ¿En qué queremos que sean competentes los estudiantes cuando aprenden ciencias naturales?

- ¿Qué competencias es posible desarrollar en los estudiantes a través de la enseñanza de las ciencias naturales?

- ¿Cómo se interrelacionarían estas competencias y valores en la formación de los estudiantes y en la práctica de los profesores?

Estas preguntas delimitan los alcances de los propósitos planteados por el proyecto y que tienen que ver con la identificación de las concepciones que acerca de competencias científicas poseen los maestros; con la determinación de estas competencias científicas que los profesores pueden orientar en sus alumnos; con el estudio de la formación en valores que los profesores proyectan en los estudiantes y en la elaboración con y desde los docentes en ejercicio de propuestas innovativas y orientadoras para el desarrollo de competencias y valores desde el Área de Ciencias Naturales y Educación Ambiental.

Los supuestos de base para esta investigación están relacionados con los siguientes aspectos:

-El trabajo conjunto de los profesores es efectivo para movilizar su pensamiento pedagógico y, consecuentemente, sus prácticas educativas "si se facilita un trabajo colectivo de una cierta profundidad en torno a problemas de interés, los profesores podemos cuestionar las concepciones y prácticas asumidas acríticamente y construir conocimientos que son coherentes con los que la literatura específica recoge como fruto de la investigación e innovación didáctica" (Gil, D. 1991). Por lo anterior, partiendo desde el saber de los maestros y brindándoles condiciones de interpretación y comprensión, se hará posible una identificación de las competencias y valores que puedan orientar en sus educandos.

- Las reformas en educación traen mejores resultados si el docente apropia el sentido e implicaciones de los nuevos enfoques. Por tanto deben darse los necesarios tiempos y condiciones para que los educadores apropien los nuevos enfoques.

- Las competencias y formación en valores en los estudiantes se lograrán si el docente que orienta su desarrollo tiene una visión clara y fundamentada sobre estos aspectos.

- Las características de los contextos nacional, regional, local e institucional, influyen en la definición de las competencias y los valores que se deben impulsar en los estudiantes.

Este proyecto de Investigación se desarrolla con el apoyo de la Universidad Pedagógica Nacional y cuenta con un equipo de investigación conformado por los autores, la profesora Lilia Reyes y las estudiantes Rosario Sarmiento, Liz Dardy Gutiérrez, y María Isabel Sierra, del Departamento de Biología; participan además, un grupo de profesores de Ciencias Naturales en ejercicio, de varias instituciones del Distrito capital. 


\section{REFERENTES}

\section{¿Cómo llega el discurso sobre competencias al contexto escolar?}

A diferencia del movimiento pedagógico de inicio de los 80's, las competencias, como una transformación educativa, no aparece como propuesta desarrollada al interior de la comunidad de educadores, sino como política estatal, relacionada más a procesos de evaluación de resultados y a medición de la calidad de la educación.

El documento marco que ha orientado la política educativa en los seis últimos años, ha sido el elaborado por Misión de Ciencia, Educación y Desarrollo, que dentro de las múltiples recomendaciones para lograr la calidad de la educación, propone fortalecer el Sistema Nacional de evaluación de la educación estableciendo la necesidad de realizar un Examen de Estado al finalizar el 9 grado de educación básica, centrado en las llamadas competencias básicas que consisten en lectura comprensiva y rápida, escritura y producción de textos, símbolos, medios y gráficos; la capacidad de expresión comunicativa escrita y distintos tipos de razonamiento o habilidades de pensamiento, como habilidad para hacer inferencias, para razonar deductiva e inductivamente, y para el pensamiento lógico matemático.

La implementación de estas políticas se inicia en la ciudad de Bogotá, donde a través de la Secretaría de Educación, se realizaron pruebas sobre competencias básicas a niños de los grados $3^{\circ}$ y $5^{\circ}$ y posteriormente a los grados $7^{\circ}$ y $9^{\circ}$, dentro del proyecto de Mejoramiento de la Calidad de la Educación, que tiene como propósito determinar los resultados de las acciones educativas; estas pruebas se aplican de manera censal y su objetivo es recoger en forma periódica información sobre los aprendizajes de los estudiantes y sobre los enfoques pedagógicos que prevalecen en las instituciones escolares 1998-2001.

Por otra parte el Ministerio de educación a través del ICFES, modifica los exámenes de estado para el ingreso a la educación superior (finales de 1998); para tal fin, éstas entidades publican una serie de folletos y libros, al igual que se realizan una serie de talleres, simposios y conferencias donde se muestran las características de la nueva prueba y de lo que significa evaluar en el modelo de las competencias. Se parte de cambiar la concepción de evaluación, entendida ahora como práctica social y cultural, que pueda suscitar la reflexión, el debate, la discusión, y la generación de otras propuestas en el contexto educativo nacional, y de proponer la concepción de ciencias naturales como construcción cultural de significado, en la idea de impulsar una visión contemporánea de estas disciplinas. Se reconoce, entonces, el carácter cultural de las ciencias entendiéndose como un campo del actuar humano construido en comunidad.

Estas nuevas pruebas surgen en el marco de un cuestionamiento a las evaluaciones centradas en el rendimiento académico, en el que se tenían en cuenta más los contenidos curriculares; ahora se pretende centrar la evaluación del logro educativo en las competencias, como alternativa para el aprendizaje significativo asociado con el uso comprensivo de sistemas simbólicos y conceptuales.

A escala internacional se presenta un movimiento alrededor del tema educativo, donde el término competencia es tratado como competencia personal en oposición al concepto de calificación profesional, dado que el progreso técnico modifica las calificaciones que requieren los nuevos procesos de producción; porque a las tareas puramente físicas, suceden tareas de producción más intelectuales, más cerebrales. Este enfoque se 
muestra en el capítulo $4^{\circ}$ del documento La Educación Encierra un Tesoro, Informe a la UNESCO de la comisión internacional sobre educación para el siglo XXI, presidida por Jacques Delors.

En el ámbito latinoamericano, es conocido el texto: Educación La agenda del siglo XXI, Hacia un desarrollo Humano, del Programa de Naciones Unidas para el Desarrollo y dirigido por Hernando Gómez Buendia, donde de manera parecida, al informe a la UNESCO, el concepto de competencia es interpretado como competencias para el trabajo, competencia laboral y es definida como la capacidad real de lograr un objetivo o resultado ocupacional en un contexto dado: una capacidad comprobada para llevar a cabo una tarea en el ámbito operacional de a ocupación respectiva. Además de los conocimientos y habilidades propios de cada caso, hoy se subraya la importancia de que el alumno comprenda lo que hace. En este texto se incluyen ejemplos de cómo para los empleos, en varios países, ya se usan estándares de rendimiento, léase competencia, para diferentes profesiones.

Por otra parte, para no quedarnos en la ingenuidad del deber ser, hoy somos conscientes del trasfondo ideológico de estas nuevas concepciones educativas, donde históricamente, por lo menos en el modernismo, los procesos educativos están ligados a los de producción y consumo, en sociedades donde la economía de mercado define, según sus intereses, modelos educativos que conllevan a imaginarios de lo que es lo humano y la educación.

Hoy estamos inmersos en la globalización del mercado y su influencia también se observa en la educación, mediante la implementación de estándares, principalmente en ¡os aspectos científicos y tecnológicos, además de maximizar capacidades intelectuales: saber hacer, entender y actuar. ¿Será este el caso de las famosas pruebas TIMSS y que nos fue tan mal?

Recientemente en Lecturas Dominicales del Tiempo, en su artículo Atraso Nacional, Significado de la Educación, Antonio Yepes, ex ministro de educación de Colombia, se pregunta ¿Qué estamos globalizando? El autor muestra cómo, en Colombia, la evolución de la educación no ha sido un verdadero propósito nacional y cómo esto conlleva implicaciones; al respecto dice: “. . . Para la mayoría de los analistas el modelo de desarrollo neoliberal es incompatible con el necesario fortalecimiento de los sectores sociales del desarrollo. Pero el Banco mundial lo mira de otra manera: El buen funcionamiento de los mercados engendra en forma usual y natural una mayor justicia social. La distancia entre las citas de los expertos y la realidad social al parecer cada vez se amplía más. El neoliberalismo privilegia al mercado sobre el estado y promueve la inserción internacional a costa de una enorme fragmentación social, considera que el crecimiento económico lleva al desarrollo y a la equidad social, posterga la solución de los problemas de desigualdad y pobreza con la ilusión de que la libre competencia da lugar a una mayor justicia social. No se debe globalizar la pobreza, el analfabetismo, el desempleo, la exclusión social. Globalicemos el bienestar. Los países desarrollados deben encausar sus esfuerzos hacia la globalización de una vida digna, que ella sea un valor para el servicio de todos." (Yepes A., 2000).

\section{CONTEXTO TEÓRICO SOBRE COMPETENCIA}

La noción de competencia fue introducida por Chomsky (1971), para explicar el carácter creativo o generativo de nuestro lenguaje y para dar cuenta de la extraordinaria 
facilidad con la que el niño se apropia del sistema lingüístico. Señala que el lenguaje refleja los procesos de la mente humana y da forma al curso y al carácter del pensamiento; ahora bien, Chomsky (1971), plantea que 'para llegar a entender cómo el lenguaje se usa o se adquiere, debemos abstraer para su estudio separado o independiente un sistema cognoscitivo, un sistema de conocimientos y creencias, que se desarrolla en los primeros años de la infancia y que opera en concurrencia recíproca con muchos otros factores determinando los tipos de comportamientos lingüístico accesibles a la observación".

Según este autor, tenemos un modelo de funcionamiento lingüístico basado en el conocimiento que los hablantes poseemos de la lengua, de tal manera que cada vez que hablamos ponernos en uso o actualizamos el conocimiento de las reglas finitas que rigen el sistema lingüístico que empleamos; ese conocimiento de carácter formal y abstracto es lo que Chomsky denomina competencia lingüística. Este conocimiento no es accesible a la conciencia de quien lo usa y solo tenemos evidencia del él a través de la actuación o desempeño lingüístico (habla, escritura, lectura).

Según lo anterior y para Torrado (1999), se pueden evidenciar los rasgos esenciales del concepto de competencias definidas como:

a) Conocimiento especializado o de carácter específico.

b) Conocimiento explícito en la práctica y de carácter no declarativo.

c) Derivado solo parcialmente de un proceso de aprendizaje, aun cuando requiere de la experiencia social y cultural.

Torrado destaca además que este concepto de competencia cobra fuerzas dentro de la Psicología Cognitiva, donde la actividad mental ya no es descrita en términos de unas supuestas aptitudes o capacidades mentales innatas, sirio que las nuevas explicaciones se centran en las operaciones que realiza la mente humana frente a determinadas tareas.

De todo esto surge el interés por comprender los desempeños o realizaciones de una persona a través de la identificación de todos los elementos que participan cuando un sujeto hace una determinada tarea. Ahora bien, el estudio sobre la actividad real del sujeto puso en evidencia la importancia del contexto en que ella se realiza.

En el campo del lenguaje Hymes (1972), introduce la idea de competencia comunicativa dinámica y diferencial, que incorpora los factores socio-culturales y reconoce el papel fundamental que tienen los elementos de la situación de comunicación en nuestra actuación lingüística. De igual manera se retoman las ideas de Vigotsky sobre el carácter situado de nuestra actividad mental, la cual es mediada y modelada por los llamados artefactos culturales (Torrado, 1999). Acá cabe destacar que la persona se concibe como un sujeto distinto, un sujeto comunicativo, que construye conocimientos que son mediados por lo social y lo cultural.

Se esbozan así dos tradiciones teóricas que miran la competencia desde sus propios referentes: la primera propone entenderla como un conocimiento actuado de carácter abstracto universal e idealizado, la segunda la entiende como la capacidad de realización, situada y afectada por el contexto en que se desenvuelve el sujeto y la actuación misma. La competencia, tal como se la mira hoy, está asociada directamente al desempeño, expresado concretamente en la manifestación de los recursos con que cuenta un individuo para realizar una tarea o actividad. (Cárdenas, 1999) 
El entender y caracterizar adecuadamente <lo que se aprende> y llega a saberse, la competencia subyacente, resulta de trascendental importancia para la comprensión del funcionamiento de la mente humana lo cual puede aportar en la tarea de construir una teoría del aprendizaje, que incluya otras áreas de la competencia humana.

El aspecto de la comprensión aparece destacado en la propuesta de Gardner (1993), quien insiste en que la escuela debe conseguir que los estudiantes se alejen del aprendizaje memorístico y alcancen una verdadera comprensión de las materias y de los conceptos con los cuales han estado trabajando, para luego poder aplicar ese saber de un modo apropiado a nuevas situaciones.

Pareciera que el interés está puesto en mejorar la educación que reciben los jóvenes colombianos, como lo decía Rodolfo Llinás (1993), "el problema es el siguiente: se enseña sin asegurarse de que entienda lo aprendido... Para que surja la pedagogía del <entender> sé requiere que a los estudiantes no sólo se les haga hincapié en la memorización, sino que se les dé el marco necesario para que lo memorizado tenga una localización en un "árbol mental" que reúna e integre el conocimiento. Que se le cree al estudiante una mente globalizada, una "cosmología general" que le permita utilizar su conocimiento".

Por otro lado, el informe a la UNESCO distingue las nociones de calificación y competencia. Pareciera que la concepción de la calificación ha hecho énfasis en las dimensiones cognitivas e informativas. Por el contrario, la noción de competencia personal "combina la calificación propiamente dicha, adquirida mediante la formación técnica y profesional, el comportamiento social, la aptitud para trabajar en equipo, la capacidad de iniciativa y la de asumir riesgos" (Delors, 1996). La formación de competencias rescata la importancia de la capacidad de comunicación, trabajar con los demás, aportar y solucionar conflictos.

De esta manera, surgen propuestas de nuevos enfoques para la educación, que apuntan a la formación basada en no sólo lograr competencias básicas en los individuos, sino también un énfasis en competencias hacia el trabajo y que intentan resolver el problema del distancia-miento entre los procesos de formación y las necesidades del sector productivo, respecto a competencias laborales "es un rumbo y un conjunto de acciones que contribuirían a desarrollar <sistemas de educación para el trabajo> que promuevan la formación y acrediten la adquisición de competencias complementados con <sistemas de información> sobre el mercado laboral y las ofertas de educación" (Gómez, et. al. 1998).

Así, la competencia laboral es definida como "la capacidad real de lograr un objetivo o resultado ocupacional en un contexto dado: una capacidad comprobada para llevar a cabo una tarea en el ámbito operacional de la ocupación respectiva. Además de los conocimientos y habilidades propios de cada caso, hoy se subraya la importancia de que el alumno comprenda lo que hace. Así la compresión es el elemento que diferencia al trabajador competente de hoy, del trabajador calificado del pasado: comprender su trabajo y el medio donde trabaja es la clave para que aporte a la solución de problemas, tenga iniciativa para resolver situaciones inesperadas y cuente con la capacidad de aprender constantemente" (Gómez et. al. 1998). En ésta propuesta se destaca que "la formación basada en competencias se conjuga muy bien con las alternativas emergentes en el mundo educativo como son el autoaprendizaje, la desescolarización, el uso de nuevas tecnologías y el aprendizaje en el trabajo (Gómez et. al. 1998). 
En un intento de síntesis podríamos decir que aunque el discurso de las competencias surge en los campos lingüístico, psicológico y productivo, su introducción en el ámbito educativo implica no sólo revisar la evaluación y sus instrumentos formales de aplicación sino remirar lo educativo en general, ligado a lo social y cultural. Su intencionalidad está centrada en las posibilidades de hacer de un sujeto en sociedad y en contextos particulares dentro de una sociedad del conocimiento y de la convivencia. Todo esto nos obliga a los maestros a hacer una reflexión antropológica, psicológica y filosófica en torno a los fines de la educación que le aporte a las personas posibilidades reales para la creación de sentidos, significados y acuerdos en grupos que se constituyen en redes de comunicación.

Indudablemente el desarrollo de competencias básicas (interpretación, argumentación y proposición), es deseable en individuos pensados como partícipes dinámicos de una sociedad de comunicación. Estas habilidades no sólo se desarrollarían en el ámbito escolar, pero una educación de calidad debería al menos considerarlas como espacio para repensar el sentido de nuestras prácticas escolares, para la discusión de lo que realmente la escuela está posibilitando en los ciudadanos. A este respecto, valdría la pena preguntarnos por ejemplo (independiente de la evaluación estatal), ¿en qué se hacen competentes nuestros estudiantes con las vivencias escolares? $Y$ si nos atreviéramos a más ¿En qué somos competentes hoy los maestros colombianos?

\section{METODOLOGÍA}

La realización de esta investigación cualitativa hace énfasis en descripción e interpretación; en tal sentido las acciones parten de una ubicación en el contexto tanto del sistema educativo, como escolar, la acción de los profesores y la interrelación con los alumnos, culminando en acciones interpretativas que dan sentido a los hallazgos de la investigación.

En términos procedimentales la ubicación en el contexto comprende actividades como: seminarios y talleres, en los que se reflexiona en conjunto investigadores y profesores sobre el estado de la situación, diseño y realización de ambientes interactivos con los participantes para la producción de información y construcción de registros; observación de la acción del profesor en el aula y entrevistas con ellos, para llegar a una producción de sentido.

Las acciones de interpretación se enmarcan en un proceso de reflexión conjunta, tanto de los investigadores como de los profesores participantes en la investigación, determinación de categorías de análisis y construcción de conocimiento que articula de manera significativa las competencias científicas y formación en valores.

A manera de síntesis podemos esquematizar el proceso metodológico (Ver Figura 1). Nótese que las fases de ubicación en el contexto e interpretación se dan simultáneamente y se interrelacionan a lo largo del proceso investigativo.

La participación de los profesores en la investigación posibilita la autoreflexión y crítica sobre sus concepciones, al tiempo que transforma sus prácticas pedagógicas, tomando en consideración su propio contexto. (Ver Figura 2). 
Figura 1. Esquema Proceso Metodológico

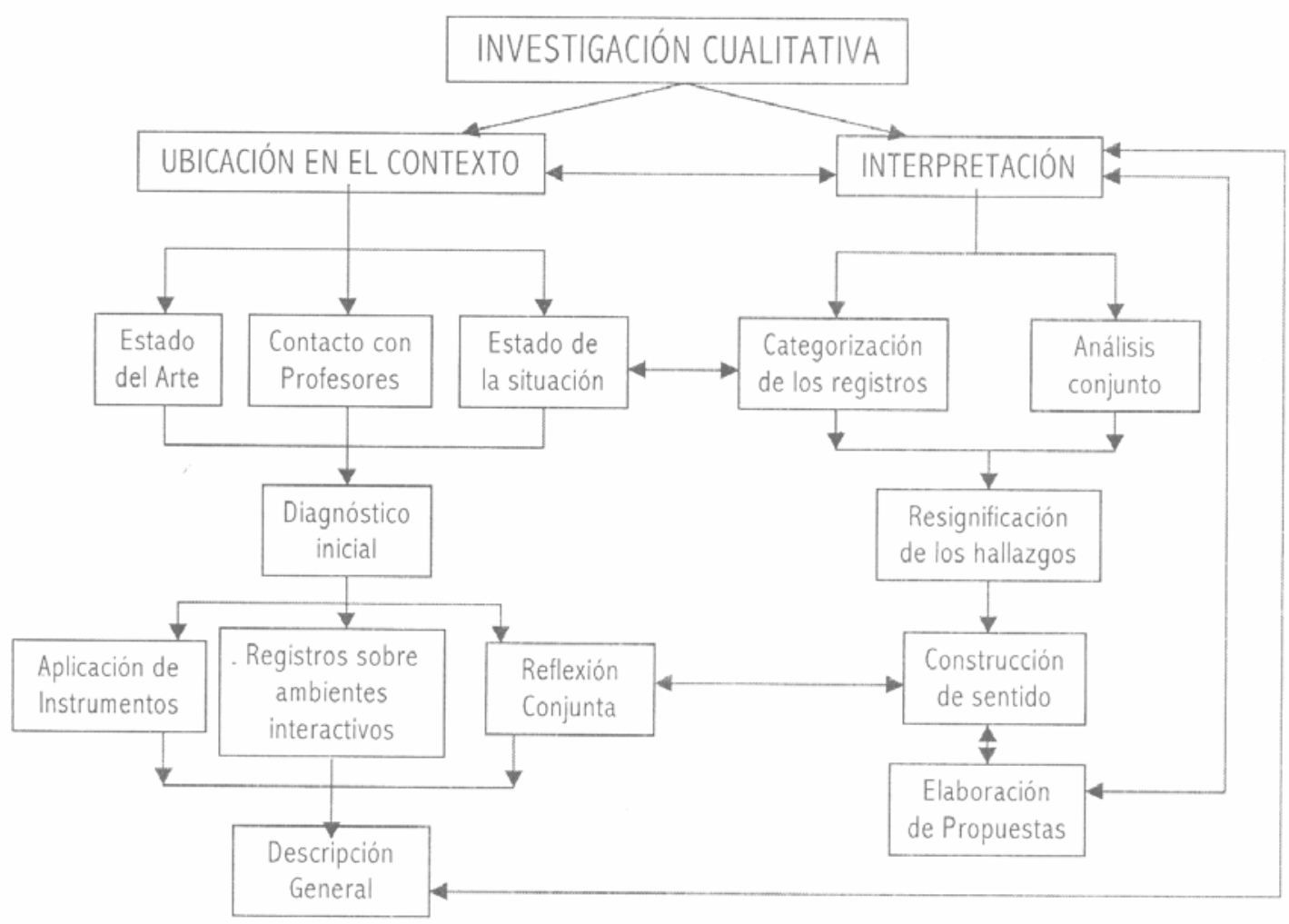

Figura 2. Transformación del docente durante el proceso investigativo

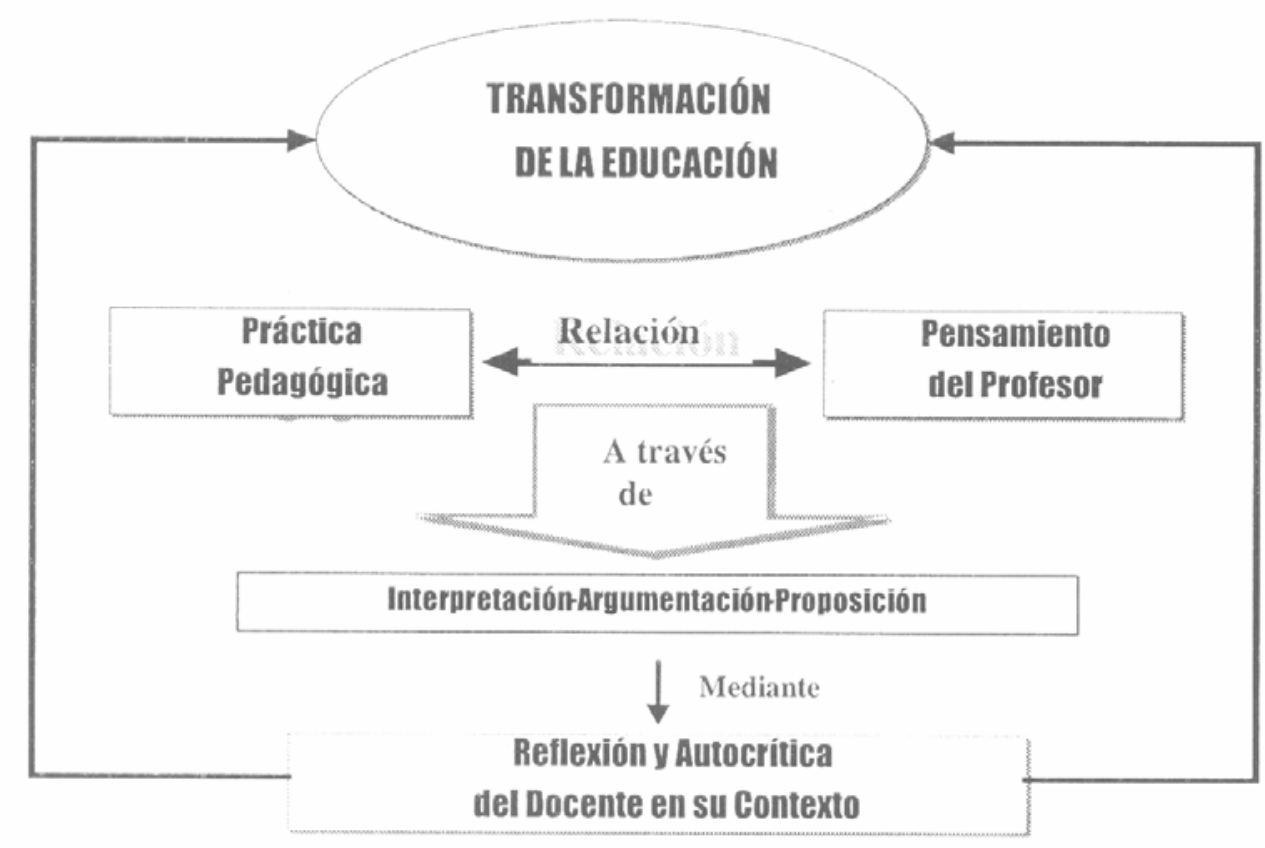




\section{RESULTADOS}

Presentaremos ahora un avance de os resultados obtenidos a partir de la interpretación de los registros de las transcripciones de los seminarios de fundamentación y talleres del equipo de investigación con profesores. La lectura y el análisis de los textos permite evidenciar que el discurso de competencias llega a los maestros, tal como se ha mostrado en la parte de referente teórico, pero hemos encontrado que cada uno de ellos lo resignifica desde su propia experiencia pedagógica, perspectiva de escuela, conocimiento, estudiante y por supuesto desde su propia mirada de sujeto.

Las concepciones de competencias evidenciadas hasta ahora en el discurso de los maestros por tanto, las podemos categorizar con el fin de profundizar en su análisis, en aspectos que tienen que ver con:

1. El maestro aborda las competencias desde la complejidad y diversidad de concepciones de conocimiento.

2. Los maestros interpretan y reconstruyen el discurso sobre competencias desde su pensamiento pedagógico.

3. Los maestros propenden por un desarrollo de competencias en el estudiante reconocido como ser integral.

4. Los maestros relacionan las competencias con lo educativo desde el marco evaluativo.

Por otro lado, el término competencia aparece citado en la Propuesta General de Examen de Estado, en el que se define como: "<Competencia es un saber hacer en contexto>, es decir el conjunto de acciones que un estudiante realiza en un contexto particular y que cumplen con las exigencias especificas del mismo. En el examen de estado las competencias se circunscribirán a las acciones de tipo interpretativo, argumentativo y prepositivo que el estudiante pone en juego en cada uno de los con textos disciplinares que hacen referencia, por su parte, al conjunto móvil de conceptos, teorías, historia epistemológica, ámbitos y ejes articuladores, reglas de acción y procedimientos específicos que corresponden a un área determinada" (MEN- ICFES, 1999), este referente ha orientado la mirada de los docentes hacia los distintos conceptualizaciones involucradas en esta definición.

Es así como notamos que la definición acerca de competencias relaciona tres conceptos importantes: EL SABER, EL HACER Y EL CONTEXTO, a los que el maestro asigna distintos sentidos según sus propios referentes.

Para ilustrar las categorías enunciadas anteriormente, mostraremos en cada tabla, algunas expresiones que a nuestro modo de ver las representan y seguidamente, nuestra interpretación.

Tabla $\mathbf{N}^{0}$ 1. Expresiones de maestros relacionadas con la categoría: el maestro aborda las competencias desde la complejidad y diversidad de concepciones de conocimiento 
"Para miel saber escomo el significado que tiene uno, el significado que yo he construido, del entorno, de mi mismo, de los demás, de los significados que me permiten a mi ubicarme para dar cuenta de algo." "Para mi el saber es un resultado. Es un producto de una actividad mental, de una actividad llamada conocer. Entonces, e/producto de conocer es el saber. Es el que circula en la información, para mi, el saber circula en la información y aparece en revistas, en videos etc." "El saber sisen las representaciones que cada uno tiene, y estoy de acuerdo con ella en que eso es muy particular y cada uno representa desde diferentes miradas." "Para mi el saber si es conocimiento, a mi me parece que el saber es como una construcción, una construcción como en un comienzo como personal pero a la vez también involucra unas interacciones con los demás individuos "'Pues para mi, el saber si es una construcción y al haber construcción con base en mis referentes estoy produciendo conocimiento." "Son mis vivencias, son mis vivencias las que me permite saber, conocer. El saber no es sólo de la escuela."

En este sentido, el maestro reflexiona y discute la concepción de saber, encontrando que respecto a ella se evidencian multiplicidad de acepciones; es decir, en los maestros hay diversidad de concepciones de conocimiento. Cabe entonces la pregunta de si tal diversidad se constituye en uñ obstáculo que habría que tratar de homogenizar, o por el contrario, tal diversidad y complejidad posibilitaría la construcción colectiva de un nuevo sentido, retomando la pluralidad de voces de los maestros.

Dentro de esta diversidad y complejidad epistemológica de las concepciones de los maestros podemos destacar las siguientes tendencias:

El saber en relación de sinonimia con el conocimiento; el saber como los significados que se tienen del entorno; el saber como un resultado, como un producto de la actividad mental, el saber como las representaciones mentales que uno tiene; el saber inscrito dentro de un mar-co individual; el saber inscrito dentro de un marco de interacción; el saber como una construcción; el saber como información. También se reconocen saberes de tipo práctico y por oposición saberes de tipo teórico y diversos tipos de saberes: escolar, científico, el saber de la cultura, el cotidiano.

Por supuesto no se trata de tipificar cada una de las anteriores concepciones en buenas o malas, positivas o negativas, por el contrario es importante explicitarlas de manera que los maestros reflexionemos acerca de ellas desde una mirada amplia, en donde desde el debate teórico, construyamos el sentido que consideramos puede aportar en la reconstrucción de la práctica pedagógica.

Tabla $\mathbf{N}^{0}$ 2. Expresiones de maestros relacionadas con la categoría: Los maestros interpretan y reconstruyen el discurso sobre competencias desde su pensamiento pedagógico 
"Que lo que uno le está enseñando del conocimiento a los conocimientos pedagógicos sea lo real, no ese puro bla bla, la carreta, sino que el muchacho viva, palpe ylo ponga en práctica." "El enseñar por competencia veo, que/o que se busca es que e/muchacho aprenda a desenvolverse en la vida cotidiana y que lo que aprenda no quede como conocimiento, sino que lo aplique en el desarrollo de lo necesite en el mundo. "Yo concluiría que la escuela no puede ser la misma, hablando desde ese discurso de/as competencias, la escuela tiene que cambiar y adaptarse, en un proceso de adaptación la escuela tiene que transformarse y el maestro tiene que transformarse ampliamente". "...y que a través de las ciencias naturales llevarles los conceptos y que esos conocimientos sean verdaderamente aplicables, que sean provechosos para el estudiante y podemos hacer muchachos adaptables..." "Yo pienso que lo miramos en el nivel de conocimientos, ya estoy asimilando y la idea de la competencia como el desarrollo de la comprensión de los conceptos" "parece que yo siempre he intentado pues los muchachos alcancen un aprendizaje significativo, es decir buscar algunas alternativas que ellos asocien lo que estamos trabajando con la realidad misma las competencias están en el marco de la discusión del aprendizaje significativo es decir, que en la medida que sea competente, está construyendo su conocimiento, es decir esto es aprendizaje significativo...

En estas expresiones de los maestres vemos como acomoda el discurso de las competencias con las elaboraciones conceptuales que ha construido a través de su formación inicial y su experiencia profesional, establece relaciones entre aquel y su práctica misma. Por tanto, para algunos maestros las competencias no las consideran nuevas dentro de su quehacer porque las justifican desde el entramado conceptual de su saber pedagógico, que es producto de la síntesis de diversas propuestas pedagógicas con las cuales ha estado en contacto. Ejemplos de ello son asimilar las competencias con el aprendizaje significativo, con la comprensión, con los proyectos de aula, etc.

Tabla $\mathbf{N}^{0}$ 3. Expresiones de maestros relacionadas con la categoría: Los maestros propenden por un desarrollo de competencias en el estudiante reconocido como ser integral

El maestro debe considerar al estudiante como un sujeto, no cualquier clase de sujeto sino un sujeto de saber". "lo que implican las competencias es que el muchacho intente, quiera, ponga entusiasmo para hacerlas cosas.. .Y cuáles son las consecuencias de eso." "Cuando en un momento dado estás tomando algo que en la vida práctica al muchacho se le va a presentar, que va a tener que aplicar ese tipo de información, que lo canalice para que lo adquiera, ese si es el desarrollo de la competencia para milo que buscan las competencias son que la persona sea la que desarrolle y se capacite para ella misma no para los demás..." "hoy le permite a uno darle importancia que no es recitar información sino que es más bien cómo esa información la utilizo yo en problemáticas que tengo, que yo construyo, que yo invento o que tengo en la vida diaria para poder actuar." 
En este sentido, el discurso de los maestros sobre competencias se ubica en un contexto concreto, la vida misma, la cotidianidad. Así el maestro reconoce que el conocimiento escolar, no puede estar al margen de la vida de los estudiantes, por el contrario los maestros asumen una postura en la cual se considera que el trabajo del aula debe servir para entender o explicarse fenómenos naturales o sociales. Sin embargo, subsisten dudas en los maestros respecto a si el discurso de competencias privilegia el contexto de la vida y la cotidianidad, o si se refiere al contexto de los discursos disciplinares.

Tabla No 4. Expresiones de maestros relacionadas con la categoría: Los maestros relacionan las competencias con lo educativo desde el marco evaluativo

Yen lo que yo más he sentido, o en/o que más me siento coda, osen la evaluación de esas competencias ..." "..Entonces yo digo a nivel general que este discurso de competencias implica una transformación en las practicas evaluatívas ¿Bueno, pero como vamos a hacer finalmente, vamos a evaluar, por logros o indicadores de logro o por competencias..? lo que yo detectaba ah,; era que las respuestas todas eran posibles, pero hay una que es más posible que las otras y esa que es más posible es la que de pronto debe medir la capacidad de interpretación, argumentación y de proposiciones Entonces van a salir muy mal evaluados en determinadas competencias cuando tienen un potencial grandísimo en otras que la prueba ha omitido.."

Los maestros asumen el discurso de competencias desde el marco evaluativo, tanto de los procesos de aprendizaje de los estudiantes, del rendimiento académico o de su propio desempeño. Esto significa que la preocupación de los maestros se inscribe dentro de la evaluación misma y no en el desarrollo de las competencias propuestas. Al igual que en otros espacios de discusión, los maestros manifiestan su preocupación por vincular el discurso de competencias con la manera de evaluar, de diseñar preguntas, de cómo verificar si se está o no desarrollando competencias en los estudiantes.

Así el maestro inscribe la categoría competencias, dentro del marco de la evaluación, es decir, no trasciende el sentido mismo de potenciar y desarrollar las competencias, por el contrario genera un vínculo directamente proporcional entre competencias y evaluación. Vale a pena señalar si respecto a las competencias pudiesen vincularse otros marcos que no necesariamente correspondan a la evaluación.

Por otra parte, se establece una relación directa con la prueba ICFES, asumiendo los maestros que la prueba en si misma no reconoce lo que se sucede en el aula. Es decir la objetividad de la prueba desconoce la subjetividad que en el aula de forma constante se manifiesta.

\section{CONSIDERACIONES FINALES}

El desarrollo de la investigación ha generado diversos hallazgos, replanteamientos de algunos aspectos de la misma, e innumerables preguntas que hacen de este trabajo un ejercicio de indagación, descripción e interpretación en espiral continuo y dinámico. 
En este sentido es importante destacar algunos hallazgos que arroja la interpretación que acerca de los textos producidos por los maestros, el grupo de investigación ha venido realizando, entiéndase que es una primera aproximación, lo que implica que tales hallazgos pueden ser reinterpretados incorporando otras perspectivas no consideradas hasta el momento.

Así podemos mencionar los siguientes aspectos:

- El pensamiento del profesor genera un proceso de mediación respecto al discurso de competencias, es decir tal discurso se convierte en plural y deja de ser direccional y unívoco.

- El significado o sentido que acerca de competencias poseen los maestros se relaciona o es consecuencia de la imagen que de conocimiento poseen los docentes, de sus referentes pedagógicos, del reconocimiento del estudiante como ser integral y fundamentalmente ligado al aspecto evaluativo.

- Con relación a la concepción de conocimiento, se plantea la discusión por ejemplo si es de orden individual o producto de interacción social, en la medida que una u otra postura define multiplicidad de eventos en la escuela. Al respecto vale la pena rescatar la discusión que plantea Torrado (1 999b) sobre "la competencia aparece como un conocimiento en acto y no tanto como un conocimiento formal $y$ abstracto'Ç tal como pensaba Chomsky; conocimiento que es situado, concreto y cambiante. Así mismo se postula un sujeto contextualizado desde el punto de vista social y por tanto particular, que se contrapone a los sujetos ideales y universales, como el hablante-oyente ideal de Chomsky $o$ el sujeto epistémico de Piaget. En este sentido, los maestros podemos retomar los postulados de Piaget y las consecuencias pedagógicas que ello conduce, acercarnos a los planteamientos de Vigotsky y su psicología cultural o podemos entrecruzar estas teorías y reconocer en ellas sus bondades para reconstruir nuestra práctica pedagógica. Lo importante entonces no es inscribirnos en una u otra teoría, lo fundamental es ser conscientes de nuestras decisiones y tratar de ser coherentes con ellas.

- Reconocer la complejidad y diversidad de las concepciones de los maestros, es un aspecto indispensable en el diseño de políticas educativas. En este sentido, es importante recalcar que la modificación de la práctica pedagógica, hacia la cualificación de la educación, se inicia en el aula, en la interacción de maestros y estudiantes y por ello retomar, explicitar y tematizar lo que ocurre allí es un aspecto fundamental si se quiere generar nuevas practicas pedagógicas.

\section{BIBLIOGRAFÍA}

Alcaldía Mayor de Santafé de Bogotá. SED. 1999. Resultados. Evaluación de competencias básicas en lenguaje, matemática y ciencias.

Cárdenas, F. 1999.Conocimientos, logros, habilidades, competencias y... ¿Qué evaluar? En: Hacia una cultura de la evaluación para el siglo XXI. Bogotá: Universidad Nacional de Colombia.

Chomsky, N .1971. El lenguaje y el entendimiento. Barcelona: Biblioteca Breve Seix. Barral. 
Chona G., Castaño N., Arteta J., Leudo M., Valencia S., Martínez S., 1998. Aproximación a las Creencias que Orientan la Práctica del Profesor de Biología. Revista de la Facultad de Ciencia y Tecnología. Universidad Pedagógica Nacional. No.4

Delors J. 1996. La educación encierra un tesoro. Informe a la UNESCO de la Comisión Internacional sobre la Educación para el Siglo XXI. Santillana Ediciones Unesco.

Gardner H. 1993. La mente no escolarizada. Cómo piensan los niños y cómo deberían enseñar las escuelas. Barcelona: Editorial Paidós.

Gil, D. 1991. ¿Qué hemos de saber y saber hacer los profesores de ciencias?. Enseñanza de las ciencias, 9 (1). Pag. 70.

Gómez H., et. al. 1998. Educación. La agenda del siglo XXI. Hacia un desarrollo humano. PNUD. Bogotá: TM Editores.

Hymes D. 1972. Acercado la Competencia Comunicativa. En: Forma y función 9 (1996), pág. 13-37 Trad. Por Juan Gómez.

Llinás R. 1993. El Reto: Ciencia, Educación y Desarrollo: Colombia en el Siglo XXI. En: Colombia: Al filo de la oportunidad. Misión ciencia, educación y desarrollo. IDEPBogotá.

Ministerio de Educación Nacional. ICFES. Examen de Estado para el ingreso a la educación superior. Cambios para el siglo XXI. Propuesta General. 1999. Bogotá

Reyes, L., Salcedo L., Perafán, G. 1999. Acciones y creencias. Tesoro oculto del educador. Tomo 1. Universidad Pedagógica Nacional. Santafé de Bogotá.

Torrado, M. C. 1999. El desarrollo de Las competencias: Una propuesta para la educación colombiana. Taller de evaluación de competencias básicas. Bogotá: Universidad Nacional de Colombia.

Torrado, M. C. 1 999b. Educar para el desarrollo de las competencias: Una propuesta para reflexionar. En: Competencias y proyecto Pedagógico. Universidad Nacional. Bogotá: Unibiblios.

Yepes, P. A. 2000. Atraso Nacional, Significado de la Educación. El Tiempo. Lecturas dominicales. Agosto 13. 